\title{
SOME EXAMPLES FROM INFINITE MATRIX RINGS
}

\author{
ROBERT W. MILLER AND DARRELL R. TURNIDGE
}

\begin{abstract}
Examples are given of the following: (1) an injective Noetherian right module which is not Artinian, (2) a linearly compact Noetherian right module over a right perfect ring which is not Artinian, and (3) a (linearly compact) Artinian left module over a right perfect ring which is not Noetherian. These examples answer questions posed by J. W. Fisher and F. L Sandomierski in the Proceedings of the Utah Conference on Ring Theory held in 1971.
\end{abstract}

The purpose of this note is to present two types of examples. The first is an example of an injective Noetherian right module which is not Artinian. In investigating chain conditions on injective modules [4], the authors were unable to locate an example of such a module in the literature. In the recent Proceedings of the Utah Conference on Ring Theory held in $1971, \mathrm{~J}$. W. Fisher poses as an open question the existence of such a module [3, p. 378].

The second type of example answers two questions raised by F. L. Sandomierski in the same Proceedings. Sandomierski shows that every linearly compact left (right) module over a right perfect ring is Artinian (Noetherian) and poses the question of whether every linearly compact left (right) module over a right perfect ring is Noetherian (Artinian) [5, Propositions 2.9 and 2.12]. Examples are given showing that in both cases the answer is no.

Throughout the following all rings have a unit element and all modules are unitary. Module homomorphisms are written opposite the scalars.

1. A non-Artinian injective Noetherian right module. Let $A$ be a division ring and let $B$ denote the ring of countably infinite column finite matrices over the ring $A$. We identify $B$ with $\operatorname{End}\left(V_{A}\right)$ for $V$ a right vector space over $A$ with countable basis $\left\{x_{i}\right\}_{i=1}^{\infty}$. Let $C$ denote the subring of $B$ consisting of all upper triangular matrices with entries from $A$. Let $\left\{e_{i j}\right\}_{i, j=1}^{\infty}$ denote the matrix units of $B$ having the unit element of $A$ in the (ij)th

Received by the editors July 17, 1972.

AMS (MOS) subject classifications (1970). Primary 16A42.

Key words and phrases. Injective modules with chain conditions, linearly compact modules with chain conditions, perfect rings.

(c) American Mathematical Society 1973 
position and zeros elsewhere and let $e_{i}$ denote the idempotent $e_{i i}$ for $i=$ $1,2, \cdots$.

Claim 1. As a left $C$-module, $V$ is flat.

Proof. For each $j=1,2, \cdots$, let $V_{i}=\sum_{i=1}^{j} C x_{i}$. Then, as a left $C$-module, $V_{i} \cong{ }_{C} C e_{i}$. Thus ${ }_{C} V_{i}$ is projective. Since ${ }_{C} V$ is the direct limit of the ${ }_{C} V_{i}$ 's, ${ }_{C} V$ is flat.

Claim 2. $B_{C}$ is injective.

Proof. Let $X_{C}$ be an arbitrary right $C$-module. By [2, Proposition VI.5.1], since $V_{A}$ is injective,

$$
\begin{aligned}
\operatorname{Ext}_{C}^{1}\left(X_{C}, B_{C}\right) & =\operatorname{Ext}_{C}^{1}\left(X_{C}, \operatorname{Hom}_{A}\left({ }_{C} V_{A}, V_{A}\right)\right) \\
& \cong \operatorname{Ext}_{A}^{1}\left(\operatorname{Tor}_{1}^{C}\left(X_{C},{ }_{C} V_{A}\right), V_{A}\right) \\
& =\operatorname{Ext}_{A}^{1}\left(0, V_{A}\right)=0 .
\end{aligned}
$$

Thus $B_{C}$ is injective.

Claim 3. The right $C$-module $e_{1} C$ is injective and Noetherian but not Artinian.

Proof. Since $B_{C}=e_{1} B_{C} \oplus\left(1-e_{1}\right) B_{C}, e_{1} B_{C}$ is injective. But $e_{1} B=e_{1} C$, so $e_{1} C_{C}$ is injective. Since every $C$-submodule of $e_{1} C$ appears in the chain $e_{1} C=e_{11} C \supsetneqq e_{12} C \supsetneq \cdots \neq \cdots$

2. Linearly compact modules over perfect rings. A ring $R$ is called left perfect if every left $R$-module has a projective cover. According to Bass $[1$, Theorem $\mathrm{P}]$, in order that $R$ be left perfect it is necessary and sufficient that $R / J$ be semisimple and $J$ be left $T$-nilpotent where $J=J(R)$ denotes the Jacobson radical of $R$.

Let $X_{R}$ be an $R$-module, $\left\{x_{i}\right\}_{I} \subseteq X$ and $\left\{x_{i}\right\}_{I}$ a set of submodules of $X$. The set of congruences $\left\{x \equiv x_{i} \bmod X_{i}\right\}_{I}$ is said to be solvable (finitely solvable), if there is a $y \in X$ (a $y_{F} \in X$ for each finite subset $F$ of $I$ ) such that $y-x_{i} \in X_{i}$ for each $i \in I\left(y_{F}-x_{i} \in X_{i}\right.$ for each $\left.i \in F\right)$. If every finitely solvable set of congruences in $X$ is solvable, then $X_{R}$ is said to be linearly compact.

The following slight modification of Bass's example of a right perfect ring which is not left perfect provides the examples required to answer Sandomierski's questions.

Let $A$ be a division ring and let $D$ denote the ring of all countably infinite upper triangular matrices over $A$ with constant entries on the main diagonal and having nonzero entries in only finitely many rows above the main diagonal. It is easily seen that $D$ is right but not left perfect with $J(D)$ consisting of those matrices in $D$ with zero on the main diagonal.

EXAMPLE i. Let $\left\{e_{i j}\right\}_{i, j=1}^{\infty}$ denote the $(i, j)$ th matrix units of $D$. Let $X_{D}=e_{11} D$. Since the chain $X_{D}=e_{11} D \underset{\neq}{\supsetneq} e_{12} D \supsetneqq \cdots \supsetneqq 0$ includes all the submodules of $X_{D}, X_{D}$ is Noetherian but not Artinian. In order to show 
that $X_{D}$ is linearly compact it is sufficient to show that any finitely solvable system of congruences $\left\{x \equiv x_{j} \bmod e_{1 j} D\right\}_{j \in J}$ where $J \subseteq\{1,2, \cdots\}$ is solvable. The finite solvability of the system implies that for $s<t$ in $J$ the first $s-1$ columns of $x_{s}$ and $x_{t}$ coincide. Thus a solution $y$ of the system is obtained by requiring the first $j-1$ columns of $y$ to coincide with the first $j-1$ columns of $x_{j}$ for $j \in J$. Thus $X_{D}$ is a linearly compact Noetherian right module over a right perfect ring which is not Artinian.

Example ii. The following example was suggested by Professor F. L. Sandomierski. For $i=1,2, \cdots$, define $f_{i}$ in $D$ to be the matrix with 1 in the $(i, i+j)$ positions, $j \geqq 1$, and zeros elsewhere. Let ${ }_{D} F$ be the left ideal of $D$ consisting of all matrices having only finitely many nonzero entries above the diagonal and zeros elsewhere. Let $Y_{i}=\left(D f_{i}+F\right) / F$ for $i=1$, $2, \cdots$, and let $Y=\bigcup_{i=1}^{\infty} Y_{i}$. For each $y \in Y$ there is an integer $k \geqq 1$ with $y \in Y_{k}, y \notin Y_{k-1}$ (say $Y_{0}=0$ ) and then $D y=Y_{k}$. Then one easily checks that the chain $0 \subsetneq Y_{1} \subsetneq Y_{2} \subsetneq \cdots \subsetneq Y$ includes all the submodules of $Y$. Thus $Y_{D}$ is an Artinian (hence linearly compact) left module over a right perfect ring which is not Noetherian.

\section{REFERENCES}

1. H. Bass, Finitistic dimension and a homological generalization of semi-primary rings, Trans. Amer. Math. Soc. 95 (1960), 466-488. MR 28 \#1212.

2. H. Cartan and S. Eilenberg, Homological algebra, Princeton Univ. Press, Princeton, N.J., 1956. MR 17, 1040.

3. R. Gordon (Editor), Ring theory, Academic Press, New York, 1972.

4. R. Miller and D. Turnidge, Co-Artinian rings and Morita duality, Israel J. Math. (to appear).

5. F. Sandomierski, "Linearly compact modules and local Morita duality," in Ring theory, Academic Press, New York, 1972, pp. 333-346.

Department of Mathematics, Kent State University, Kent, Ohio 44240.

Current address (Robert W. Miller): Department of Mathematics, College of William and Mary, Williamsburg, Virginia 23185

Current address (Darrell R. Turnidge): Department of Mathematics, Kent State University, Kent, Ohio 44242 